\title{
A Recognition Method of RMB Numbers Based on Character Features
}

\author{
Xuejiao Zhu \\ School of Computer Science and Engineering \\ Nanjing University of Science and Technology \\ Nanjing, China \\ zhuxuejiao1013@163.com
}

\author{
Mingwu Ren \\ School of Computer Science and Engineering \\ Nanjing University of Science and Technology \\ Nanjing, China \\ renmingwu@sina.com
}

\begin{abstract}
According to the features of RMB banknote images, combining digital image processing and pattern recognition, this paper proposes a recognition method of $R M B$ word numbers based on character features. The recognition algorithms include: image preprocessing, number region location, feature extraction and character recognition. The experimental results show that the automatic number identification system based on this method achieves high recognition rate and processing speed.
\end{abstract}

Keywords-RMB numbers; character segmentation; feature extraction ; character recognition

\section{INTRODUCTION}

RMB is the legal currency of China, and every RMB banknote has a unique crown word number, serving as the identity of it. Through the establishment of the banknotes' number sequence file, the circulation of the banknotes in the financial market can be monitored and tracked, and crimes like currency counterfeiting, robbery, stealing can be effectively controlled. Therefore, it is of great importance and application prospect to do research on the identification of RMB word numbers. Recently, many banks have applied this technology on their ATMs, identifying and recording the numbers of the RMB banknotes into and out of ATMs. In this way, banks can prevent receiving counterfeits and customers who get counterfeits from the ATMs would also be compensated.

This paper studies on the identifying process of RMB numbers and proposes a method based on character features. By optimizing several algorithms, it achieves high recognition rate.

\section{DESIGN OF THE SYSTEM}

The automatic RMB number identification system consists of following modules: image preprocessing module, number region location module, character segmentation module, feature extraction module and character recognition module ${ }^{[1][3]}$. The whole process is shown in Fig.1.

From Fig.1, it can be seen that the result of former modules would certainly affect the following modules, and finally affect the recognition of the RMB numbers. So, it's of high importance to choose a fast and efficient algorithm in dealing with every module.

In order to achieve high recognition rate and speed, we optimized some algorithms and got quite good results.

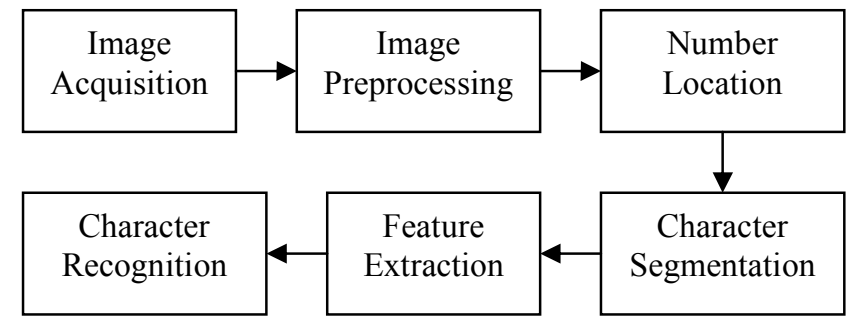

Figure 1. Flow chart of the system

\section{IMPLEMENTION OF THE SYSTEM}

\section{A. Image Preprocessing}

Image preprocessing is an important module for image recognition; it means to conduct several specific operations on the image to reach a certain goal.

- Gray scaling

First of all, convert the RGB banknote image acquired into a grayscale image. In this way, the workload can be greatly reduced, and the system becomes faster. As is known, a RGB image has three channels, namely, R, G, B channels. Every pixel of the image has these three channels and needs three bytes to be stored in the memory. While a grayscale image only contains intensity information and every pixel only needs one byte to be stored, so after gray scaling, the image needs less memory. The converting formula is shown in (1):

$$
\text { Gray }=0.299 * R+0.587 * G+0.144 * B
$$

- Image De-noising

In the process of acquiring RMB banknotes images, noises occur due to various reasons and will influence later procedures. So image de-noising is needed here. Normally, image de-noising methods are mainly divided into tow categories, namely, neighborhood-based (space domain) computing methods and transforming domain-based (frequency domain) methods. And the most commonly used methods are based on space domain, such as mean filtering, median filtering, and extreme filtering and so on. Compared with mean filtering, median filtering is not likely to cause image blur and has 
good noise immunity, so the system uses a $5 \times 5$ median filter to remove the noises.

- Image Binarization

In digital image processing, binarization is a key procedure which can greatly influence the final result. Binarization processing is to separate the region of interest (ROI) from the background in the image, in order to get the target information while discard useless information ${ }^{[7]}$. In this way, it reduces the image information to be processed, thus simplifies later processings.

Generally, an appropriate threshold is needed in binarization to convert an original image with 256

( $0 \sim 255$ ) gray levels into one with only 2 gray levels.

As is shown in (2), suppose $\mathrm{T}$ is the selected threshold, and set the background white while the target black.

$$
G(x, y)=\left\{\begin{array}{l}
255, f(x, y) \geq T \\
0, \quad f(x, y)<T
\end{array}\right.
$$

The result of binarization depends on T. Generally, the most widely used threshold selecting methods include global threshold method and local threshold method. The former considers information of the whole image while the latter divides the image into several sub-areas and selects an appropriate threshold for them in order to get the ROI information.

By observing and analyzing the RMB banknote images, it's learnt that the target area accounts for a certain proportion in the image (in this system, the proportion is around 0.06 ). So a threshold method based on proportion is proposed as is shown in (3).

$$
G(x, y)=\left\{\begin{array}{l}
255, \text { others } \\
0, \quad \text { sum }<0.06 * \text { Im gSize }
\end{array}\right.
$$

In (3), sum $=\sum_{g_{\min }}^{g} h(g), g_{\text {min }}$ and $g_{\text {max }}$ stand for the minimum and maximum gray-level of the image respectively while $h(g)$ stands for the total number of the pixels whose gray-level is $g$. Fig.2 and Fig.3 respectively show the image before and after binarization.

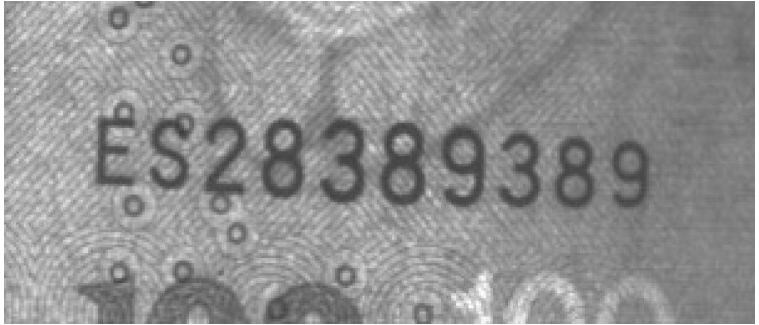

Figure 2. Image before binarization

$$
\sigma^{-}
$$

\section{ES28389389}

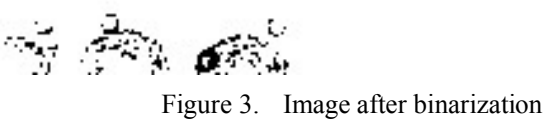

\section{B. Number Region Location And Tilt Corrction}

- Number Region Location

Projection method is used here to obtain the number region. And there are horizontal and vertical projections. Horizontal projection calculates the total number of black pixels per column while vertical projection calculates the total number of black pixels per row, as shown in (4) and (5) ${ }^{[4]}$.

$$
\begin{gathered}
P_{h}(x)=\sum_{y=0}^{\text {heihgt }} f(x, y) \\
P_{v}(y)=\sum_{x=0}^{\text {width }} f(x, y)
\end{gathered}
$$

The upper and lower boundaries of number region can be obtained through the vertical projection while the left and right boundaries could be got through the horizontal projection. After many experiments, it's found that the result is not as good as expected because of the noises that still exist in the binary image. So, some measures should be taken in order to get better results. Here to limit the size, or the length and height of the projection area can get good result as the projection area of the noises is smaller than that of the number after all. In detail, when seeking for the upper and lower boundaries, the restriction lies in that the projection size must be the largest; when seeking for the left and right boundaries, set the space between two adjacent characters $\Delta d=25$ pixels, and the projection size of every character $s u m=30$ pixels. 
- Tilt Correction

During the acquisition of RMB banknote images, tilt may occur as the camera and the banknote are not parallel and thus the number region also tilts which would affect the final recognition. So tilt correction is necessary here and easy to implement. First find the tilt degree $\alpha$ through the horizontal and vertical distances between the first and last characters of the number region respectively. Then use (6) to correct the tilt.

$$
\left\{\begin{array}{l}
x^{\prime}=x \cos \alpha-y \sin \alpha \\
y^{\prime}=x \sin \alpha-y \cos \alpha
\end{array}\right.
$$

Here, $x^{\prime}, y^{\prime}$ stands for the $\mathrm{X}$-axis and $\mathrm{Y}$-axis value of the pixel after correction respectively.

- The results after number region location and tilt correction are shown in Fig.4.

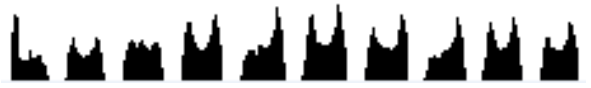

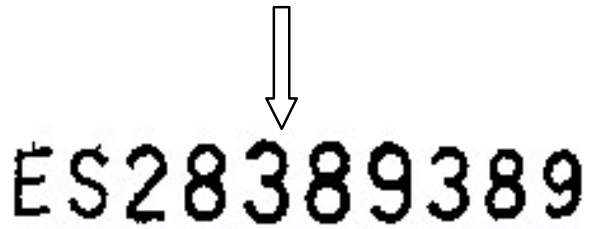

Figure 4. Result of number region location and tilt correction

\section{Character Segmentation And Normalization}

\section{- Character Segmentation}

The purpose of character segmentation is to get every single character of the numbers as the data source for the final recognition. Horizontal projection method is widely used here. Yet errors often occur in the segmentation when there are adhesive or broken characters in the image. So prior knowledge, namely the distance between every two adjacent characters and the average width and height of the all characters, is applied here to improve horizontal projection method ${ }^{[5]}$.

Through analyzing RMB banknote images, here in this system, the distance between two adjacent characters is set $\Delta d=10$, while the average width of all characters is set $\Delta w=25$. Fig. 5 shows the result.

\section{||||$|2| 8|3| 8|9| 3|8| 9 \mid$}

Figure 5. Result of character segmentation
- Normalization

Now, every single character is got with different locations and sizes which is not conducive to later processing. So normalization is proposed here.

a) As for location normalization, first set the target location, then through translation can easily locate the character at the target position ${ }^{[8]}$.

b) As for size normalization, first set the target size, here set $s=32 \times 40$ pixels per character. Then calculate the linear scaling factor according to the original and target size of a character. In the end, use nearest-neighbor interpolation algorithm to implement the scaling. The result is shown in Fig.6.

\section{$|\mathrm{E}| \mathrm{S}|2| 8|3| 8|9| 3|8| 9$ \\ Figure 6. Result of normalization}

\section{Feature Extraction}

The basic task of feature extraction is to find out those features which can best represent for the character. Some features widely used include grid feature, moment feature, and contour feature and so on. And this system proposed an 8-direction gradient feature extraction method.

As is known, edge information can be used to distinguish a character and the edge is where the gray level changes greatly in an image. Since the gradient features of the pixel distribution can reflect the changes of gray levels in the image, it's feasible to extract the gradient features of the characters for the final recognition.

First of all, apply Sobel operator to get the gradient feature of every character belonging to the number region. Then, for each character, decompose the gradient feature of every pixel onto eight directions according to standard chain code direction as is shown in Fig.7. Thirdly, divide every character into $8 \times 8$ grids, and for each grid, sum up the gradient value in eight directions respectively of all 64 pixels, thus the gird get an 8-dimensional feature. Then a character get a $(n \times 8)$-dimensional feature. (Here, $n$ means the total grid number of a character, and in this system, as each character is normalized to $32 \times 40$ pixels, so $n=(32 / 8) \times(40 / 8)=20)$. And in the end, save the feature into a template library built for the corresponding character.

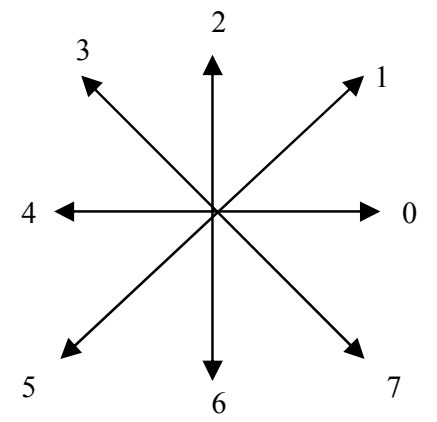

Figure 7. Standard chain code directions 


\section{E. Character Recogniton}

There are many algorithms to implement character recognition, such as template matching, nearest neighbor method (K-Nearest Neighbor), SVM (Support Vector Machine) and so on ${ }^{[2][6]}$. And here nearest neighbor algorithm is used and the final result in shown in Fig. 8.

\section{ES28389389}

Figure 8. Result of character recognition

\section{EXPERIMENTS AND CONCLUSION}

The result of experiments is shown in Table I.

TABLE I. RESULT OF EXPERIMENT S

\begin{tabular}{|c|c|c|c|}
\hline & $\begin{array}{c}\text { Training } \\
\text { Samples }\end{array}$ & $\begin{array}{c}\text { Test } \\
\text { Samples }\end{array}$ & $\begin{array}{c}\text { Recognition } \\
\text { Rate (\%) }\end{array}$ \\
\hline $\begin{array}{c}\text { English } \\
\text { Letter }\end{array}$ & 500 & 100 & 97.1 \\
\hline $\begin{array}{c}\text { Number } \\
\text { Character }\end{array}$ & 600 & 100 & 98.3 \\
\hline Total & & & 95.2 \\
\hline
\end{tabular}

Although some algorithms are optimized in they system, the recognition rate is still below $100 \%$. After analyzing the whole process of the experiments, it's found that several factors below could lead to wrong recognition:
- A RMB banknotes itself being wrinkled, contaminated during daily use;

- $\quad$ Similar characters like 1 and I, 0 and O;

- Training library not big enough.

So, in the further study, efforts can be paid into these factors in order to improve the recognition rate.

\section{REFERENCES}

[1] Hu Ming-Kuei, "Visual Pattern Recognition by Moment Invariants", IEEE Transactions on Information Theory, vol.1, pp. 179-187,1962.

[2] He Chao, Girolami M and Ross G, "Employing optimized combinations of one-class classifiers for automated currency validation", Pattern Recognition, vol. 37, pp.1085-1096, June, 2004

[3] Jinghong Duan, and Dan Luan. "Research on an Automatic Number Recognition Method for RMB Banknotes", COMPUTER ENGINEERING \& SCIENCE, vol 30, pp.66-68, 2008.

[4] J.X. Dong, A. Krzyzak, and C.Y. Suen, "A multi-net learning framework for pattern recognition", Proceedings of the Sixth International Conference on Document Analysis and Recognition, Seattle, 2001, pp.328-332.

[5] Jin Liang, and Zhiqiang Bao, "Extraction and identification of RMB crown word numbers", Modern Electronics Technique, vol.36, June, 2013, pp.70-74.

[6] Dongjiao Zhang, and ZaoBin, Gan, "Research on RMB Currency Number Identification System Based on Template Matching", Computer \& Digital Engineering, vol.40, 2012, pp. 104-108.

[7] Siqi Han, and Lei, Wong, "A Survey of Thresholding Method for Image Segmentation", System Engineering and Electronics, vol. 6, 2002, pp. 91-94.

[8] Alessandro Vinciarelli, and Juergen Luettin, "A New Normalization Technique for Cursive Handwritten Words", Pattern Recognition Letter, 2001, pp:1043 1050. 